\title{
Numerical Analysis and Verification of the Gas Jet from Aircraft Engines Impacting a Jet Blast Deflector
}

\author{
Fu-Dong Gao* D, De-Xin Wang, Hai-Dong Wang and Ming-Ming Jia
}

\begin{abstract}
The process of the gas jet from aircraft engines impacting a jet blast deflector is not only a complex fluid-solid coupling problem that is not easy to compute, but also a safety issue that seriously interferes with flight deck environment. The computational fluid dynamics (CFD) method is used to simulate numerically the impact effect of gas jet from aircraft engines on a jet blast deflector by using the Reynolds-averaged Navier-Stokes (RANS) equations and turbulence models. First of all, during the pre-processing of numerical computation, a sub-domains hybrid meshing scheme is adopted to reduce mesh number and improve mesh quality. Then, four different turbulence models including shear-stress transport (SST) $k-w$, standard $k-w$, standard $k-\varepsilon$ and Reynolds stress model (RSM) are used to compare and verify the correctness of numerical methods for gas jet from a single aircraft engine. The predicted values are in good agreement with the experimental data, and the distribution and regularity of shock wave, velocity, pressure and temperature of a single aircraft engine are got. The results show that SST $k$ - $w$ turbulence model is more suitable for the numerical simulation of compressible viscous gas jet with high prediction accuracy. Finally, the impact effect of gas jet from two aircraft engines on a jet blast deflector is analyzed based on the above numerical method, not only the flow parameters of gas jet and the interaction regularity between gas jet and the jet blast deflector are got, but also the thermal shock properties and dynamic impact characteristics of gas jet impacting the jet blast deflector are got. So the dangerous activity area of crew and equipments on the flight deck can be predicted qualitatively and quantitatively. The proposed research explores out a correct numerical method for the fluid-solid interaction during the impact process of supersonic gas jet, which provides an effective technical support for design, thermal ablation and structural damage analysis of a new jet blast deflector.
\end{abstract}

Keywords: Aircraft engine, Gas jet, Computational fluid dynamics, Jet blast deflector, Impact effect

\section{Introduction}

The high-temperature and high-speed gas jet from aircraft engines is in an over expansion state when the aircraft engines are in full afterburner state to takeoff, which has a serious impact on the flight deck due to the complicated physical phenomena such as shock wave, noise and gas tempering caused by the interaction between gas jet and the jet blast deflector $[1,2]$. The gas jet from aircraft engines has basic characteristics of supersonic flow and complex wave system. When the gas jet impacts a jet

*Correspondence: gaofudong2005@163.com

Department of Carrier Aviation Security and Station Management, Naval Aeronautical University, Qingdao 266041, China blast deflector, the thermal shock and dynamic impact will lead to thermal ablation and structure damage. The safety limits for pressure and temperature of the gas jet need to be considered due to the relatively small flight deck for carrying and taking off. Therefore, accurate analysis and control of the gas jet from aircraft engines are key steps for the design and layout of new jet blast deflectors.

At present, domestic and foreign scholars mainly use experimental method, analytical method and numerical method to carry out impact effect research of gas jet. The experimental method mainly uses a small scale model as the experimental object based on the similarity principle. The ideal results can be obtained by 
testing techniques such as PIV and LDV. However, it is difficult to obtain accurate data in the case of complicated geometry and the contradiction between geometric similarity and dynamic similarity. Furthermore, the experimental method has the problem of long cycle and high cost, which is also easy to be affected by scale effect, external interference and other factors. It is difficult to reflect the true situation of gas jet [3, 4]. At present, the widely used analytical method like one-dimensional/quasi one-dimensional is to establish a physical model and a mathematical model of gas jet by simplifying and assuming the limits of solid boundary. It ignores turbulent effect, flow viscosity and wall interference of gas jet, which makes a large deviation between computational results and experimental data [5-7]. In contrast, using a numerical method based on RANS equations to compute the impact effect of gas jet is more effective, and more detailed flow field information can be obtained [8].

Because supersonic gas jet has the characteristics of high temperature, high pressure and high speed, there are two main difficulties in numerical computation. One is that the numerical computation of supersonic gas jet with complicated geometric model and flow characteristics is sensitive to initial field. The initial deviation from actual physical field will cause difficulty of convergence, even divergence at the beginning of computation. The other is that a high-precision discrete format, appropriate turbulence model and boundary conditions are needed to capture shock wave and expansion wave of the flow field, which leads to a long computation time and large memory usage. Therefore, domestic and foreign scholars usually use two-dimensional numerical simulation model to carry out the research [9-11]. But the flow information of two-dimensional numerical results is limited, which is unable to analyze the anisotropic impact effect of gas jet on a jet blast deflector. Three-dimensional numerical model was used by some scholars to simulate the impact process of gas jet, but the thermal shock effect of gas jet was not considered [12, 13]. According to the geometry of a single aircraft engine and the characteristics of gas jet, numerical simulation of the gas jet is made combined with different turbulence models. The high-precision results with SST $k-w$ turbulence model are analyzed and verified. Then a scheme of sub-domains hybrid mesh is designed based on three-dimensional numerical model with coupling aircraft engines and a jet blast deflector. The parameters such as shock wave, velocity, pressure and temperature are got, and impact effect of the gas jet from aircraft engines on a jet blast deflector and the surrounding environment is analyzed.

\section{Numerical Computation Model}

\subsection{Mathematical Model}

The gas jet is assumed to be compressible Newtonian fluids. The governing equations are written for the mass, momentum and energy conservation, such that

$$
\begin{aligned}
& \frac{\partial \rho}{\partial t}+\frac{\partial}{\partial x_{i}}\left(\rho u_{i}\right)=0, \\
& \frac{\partial}{\partial t}\left(\rho u_{i}\right)+\frac{\partial}{\partial x_{j}}\left(\rho u_{i} u_{j}\right) \\
& \quad=-\frac{\partial p}{\partial x_{i}}+\frac{\partial}{\partial x_{j}}\left[\mu\left(\frac{\partial u_{i}}{\partial x_{j}}+\frac{\partial u_{j}}{\partial x_{i}}-\frac{2}{3} \delta_{i j} \frac{\partial u_{l}}{\partial x_{l}}\right)\right] \\
& \quad+\frac{\partial}{\partial x_{j}}\left(-\rho \overline{u_{i}^{\prime} u_{j}^{\prime}}\right)+\rho f_{i}, \\
& \frac{\partial}{\partial x_{j}}\left(\rho u_{j} H\right)=-\frac{\partial q_{j}}{\partial x_{j}}+\frac{\partial}{\partial x_{j}}\left(\tau_{i j} u_{j}\right)-\frac{\partial}{\partial x_{i}}\left(J_{i j} h_{i}\right) \\
& +S_{a}+\rho f_{i} u_{i},
\end{aligned}
$$

where $\rho$ is the gas density, $t$ is the computation time, $u_{i}$ and $u_{i}$ are velocities in the Cartesian coordinate system, $\overline{u_{i}^{\prime} u_{j}^{\prime}}$ is Reynolds stresses, $\mu$ is the molecular viscosity, $p$ is the static pressure, $f_{i}$ is the unit mass force, $\delta_{i j}$ is Kronecker delta, $h_{i}$ is the static enthalpy, $H$ is the total enthalpy, $J_{i j}$ is the diffusion mass flux, $S_{a}$ is the external heat source caused by surface reaction and radiation, $\tau_{i j}$ is the viscous stress tensor, $q_{j}$ is the heat flux.

The $k-\varepsilon, k-w$ and RSM turbulence models are used for turbulence closure, which express $-\rho \overline{u_{i}^{\prime} u_{j}^{\prime}}$ with loworder correlative term or time-average expression [14, $15]$. The $k-\varepsilon$ and $k-w$ models based on isotropic turbulence assume that the change rate of Reynolds stress and time-averaged velocity field is linear. Moreover, the concept of eddy viscosity or turbulent viscosity is introduced, so there is a big error in the computation of inhomogeneous turbulence like swirling. SST $k-w$ model has different turbulence constants, which not only increases a horizontal dissipation derivative but also takes into account the transport process of turbulent shear stress in the definition of turbulent viscosity compared with standard $k-w$ model. These features make SST $k$ - $w$ model have a wider applicable scope such as the flow with adverse pressure gradient, aerofoil, transonic shock wave, etc. [16]. RSM should have better potential to predict the key features of rotating flows than other models in theory as it considers the convection and diffuse effect of Reynolds stresses based on turbulence anisotropy, but its precision is limited to closed form so that RSM does not show the advantages in numerical computation of gas jet [17]. According to 
the Boussinesq's assumption, The SST $k$ - $w$ turbulence model can be written in Cartesian tensor form as

$$
\left\{\begin{array}{c}
\frac{\partial}{\partial t}(\rho k)+\frac{\partial}{\partial x_{i}}\left(\rho k u_{i}\right)=\frac{\partial}{\partial x_{j}}\left(\Gamma_{k} \frac{\partial k}{\partial x_{j}}\right)+G_{k}-Y_{k}, \\
\frac{\partial}{\partial t}(\rho w)+\frac{\partial}{\partial x_{i}}\left(\rho w u_{i}\right)=\frac{\partial}{\partial x_{j}}\left(\Gamma_{w} \frac{\partial w}{\partial x_{j}}\right)+G_{w}-Y_{w}+D_{w},
\end{array}\right.
$$

where $k$ is the turbulence kinetic energy, $w$ is the specific dissipation rate, $\Gamma_{k}$ is the effective diffusivity of $k, \Gamma_{w}$ is the effective diffusivity of $w, G_{k}$ is the generation of $k$ due to mean velocity gradients, $Y_{k}$ is the dissipation of $k$ due to turbulence, $Y_{w}$ is the dissipation of $w$ due to turbulence, $D_{w}$ is the cross-diffusion term.

The specific expressions above are written as

$$
\left\{\begin{array}{l}
\Gamma_{k}=\mu+\frac{\mu_{t}}{\sigma_{k}}, \Gamma_{w}=\mu+\frac{\mu_{t}}{\sigma_{w}}, \\
G_{k}=\min \left(\mu_{t} S^{2}, 10 \rho \beta^{*} k w\right), G_{w}=\frac{\alpha}{v_{t}} \mu_{t} S^{2}, \\
Y_{k}=\rho \beta^{*} k w, Y_{w}=\rho \beta_{i} w^{2} \\
D_{w}=2\left(1-F_{1}\right) \rho \sigma_{w, 2} \frac{1}{w} \frac{\partial k}{\partial x_{j}} \frac{\partial w}{\partial x_{j}},
\end{array}\right.
$$

where $\sigma_{k}$ is the turbulent Prandtl number for $k, \sigma_{w}$ is the turbulent Prandtl number for $w, \mu_{t}$ is the turbulent viscosity, $S$ is the strain rate magnitude, $F_{1}$ is the blending function, $\beta^{\prime \prime}, \beta_{i}, \sigma_{w, 2}$ are constants.

The size of the computational domain is $25 \mathrm{~m} \times 15.540 \mathrm{~m} \times 35.992 \mathrm{~m}$, and the boundary conditions include inlet, outlet and wall, as shown in Figure 1 . The specific settings are as follows:

(1) Pressure inlet: on the inlet of twin engines, total pressure $(371425 \mathrm{~Pa})$ and total temperature (2200 K) based on Boussinesq's assumption are imposed with considering the influence of wind speed. Moreover, on the section outside the outlet of engine nozzles, environmental pressure (101325

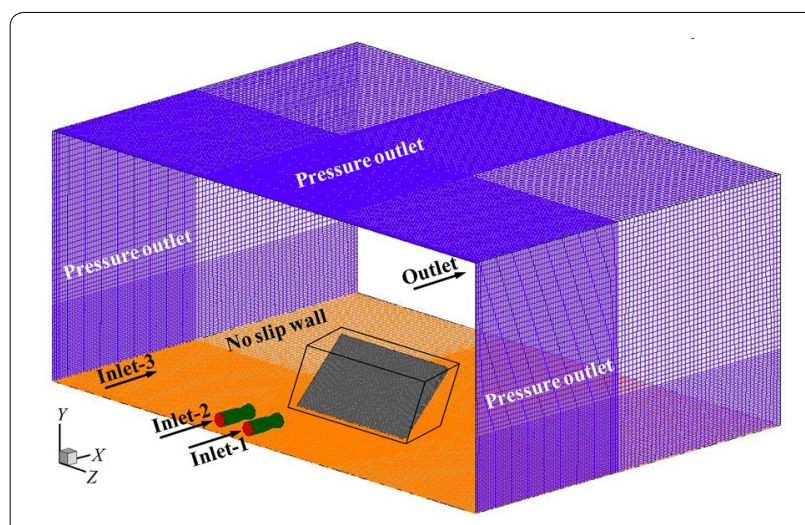

Figure 1 Computational domain of gas jet from aircraft engines
$\mathrm{Pa})$ and environmental temperature $(300 \mathrm{~K})$ are imposed.

(2) Pressure outlet: on the radial section of the computational domain and the axial section that $25 \mathrm{~m}$ away from the outlet of engine nozzles, static pressure distribution (101325 Pa) and environmental temperature $(300 \mathrm{~K})$ are imposed.

(3) Wall boundary: on the double engine nozzles, jet blast deflector and flight deck, no slip wall is imposed with considering viscous effect, and heat transfer coefficient is given.

The definition of the coordinate system is that $X$ axis points downstream, $Y$ axis points upwards and $Z$ axis accords with the right hand principle. The coordinate origin is the intersection of engine nozzles' outlet section, flight deck and starboard side of computational domain.

\subsection{Computational Model}

A carrier aircraft usually adopts two turbofan engines. A turbofan engine is installed fans and an outer duct on the basis of a turbojet engine, which has the characteristics of large thrust, low fuel consumption, low noise and long service life. The twin-engine nozzles of a certain carrier aircraft are used as the source of gas jet in this paper. The computational model of a single engine is shown in Figure 2 [18].

The jet blast deflector device is one of the key equipments to ensure the carrier aircrafts take-off safely and continuously on the aircraft carrier. It makes the hightemperature, high-pressure and high-speed gas jet from aircraft engines upward or outboard, which ensures safety of the rear crew, carrier aircrafts and equipments on the flight deck $[19,20]$. A certain jet blast deflector is used as the computational model in this paper, whose deflector assembly is composed of a series of cooling panels and a base plate component, as shown in Figure 3. The distance between engine nozzles and flight deck is $1957 \mathrm{~mm}$, and the distance between twin scramjet nozzles is $2400 \mathrm{~mm}$. When the engine is in full afterburner

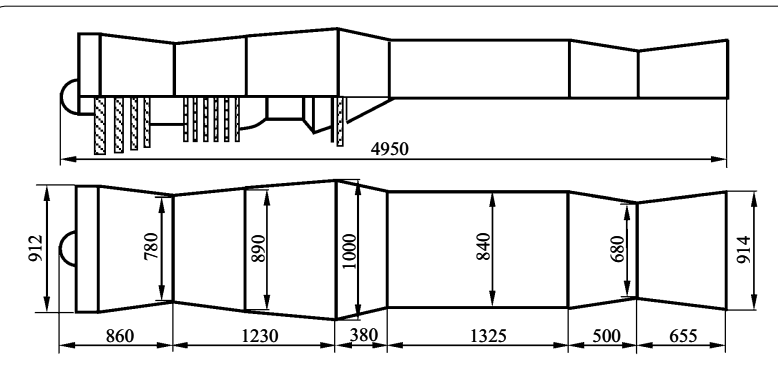

Figure 2 Computational model of a single engine 


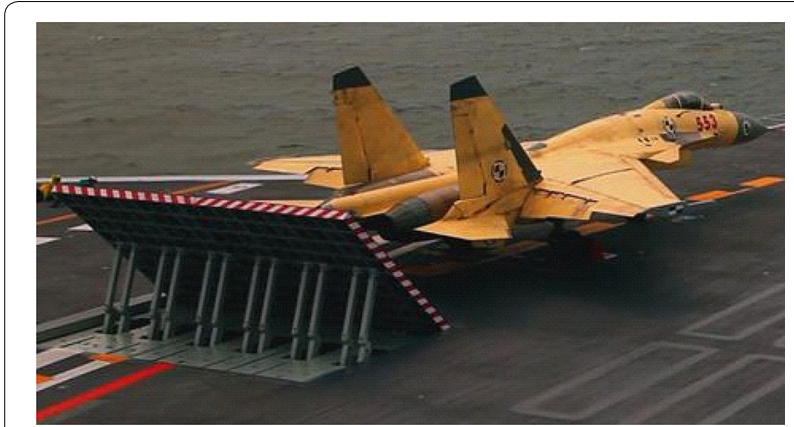

Figure 3 Computational model of a jet blast deflector

state, the pressure of nozzles inlet is $270100 \mathrm{~Pa}$, and the temperature is $2200 \mathrm{~K}$. The coordinate position of the jet blast deflector in computational domain is that $X$ axis of $5 \mathrm{~m}, Y$ axis of $0 \mathrm{~m}$, and $Z$ axis from $13.497 \mathrm{~m}$ to $22.495 \mathrm{~m}$.

\section{Mesh Generation and Numerical Method}

\subsection{Mesh Generation Scheme}

The quality of mesh directly determines the convergence, efficiency and accuracy of numerical computation. Therefore, a reasonable mesh should be generated based on the physical characteristics of flow field [21]. The computational domain is divided into three regions according to the geometry of aircraft engines and the jet blast deflector, where size functions are used to control mesh density. Twin engines' shells are defined as the boundary of respective internal flow field and each internal computational domain is filled with hexahedral cells. The prism cells are used as boundary layers on the inner surface of engines' shells. Then a cuboid is founded to enclosure the jet blast deflector and filled with tetrahedral cells. The surface of the jet blast deflector is filled with prism cells. Then the remaining outside flow field are divided and filled with hexahedral cells. Pyramid cells are used to achieve docking between the inside and the outside of the cuboid equably. The sub-domains hybrid mesh not only adjusts complicated shape of the jet blast deflector, but also improves mesh precision and reduces mesh number. In order to simulate hydrodynamic characteristics of the gas jet accurately, mesh adaptive technology is adopted in computation process, and the mesh is optimized based on total pressure gradient value for two times [22]. The surface mesh of aircraft engines and the jet blast deflector is shown in Figure 4.

\subsection{Numerical Computation Method}

The mathematical model of gas jet is a series of nonlinear equations, which employs a cell-centered finite volume method that allows the adoption of computational elements with arbitrary polyhedral shape. The

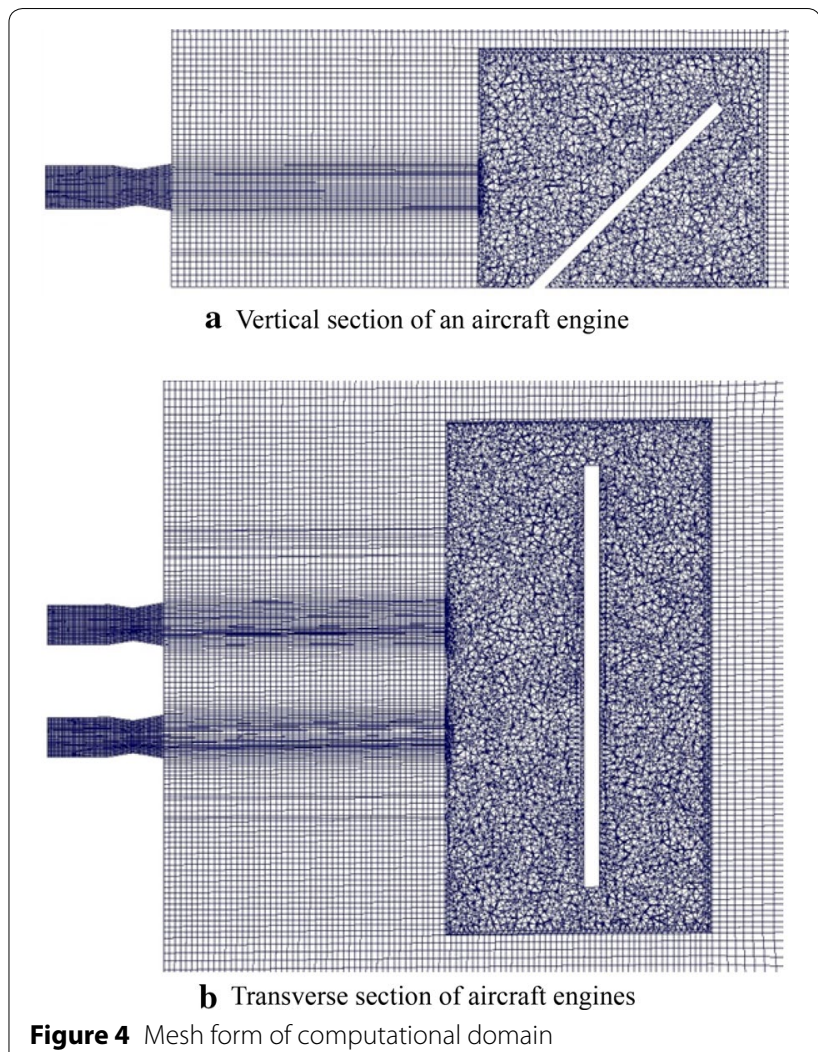

flow equation, turbulent kinetic energy equation and turbulent dissipation rate equation are discretized with second-order upwind scheme. The coupled implicit algorithm is used to solve the continuity equation, momentum equation and energy equation synchronously during the discrete process. The discrete algebraic equations are solved by Gauss-Seidel method [23]. According to the linear stability theory, Courant number is adjusted from 0.5 to 4 continuously with iterative number increasing. Algebraic Multi-Grid (AMG) method is used to accelerate the speed of convergence, and the convergence criterion is set as 0.0001 .

The supercomputer in National University of Defense Technology was used to complete the numerical simulation due to large size computational model, too much mesh and large memory occupied by coupled implicit algorithm. A total of 9 nodes including 72 cores were used in each computational process, which took 9 hours to get each converged result.

\section{Numerical Computation Results and Analysis 4.1 Verification of Numerical Method for the Gas Jet from an Aircraft Engine}

The aircraft engine mainly has full afterburner state, transition state, rated state and idle state, where the speed, pressure and temperature of its nozzle in full afterburner 
state are the maximum and the computation conditions are the most complicated [24]. In order to verify the validity of numerical method, four turbulence models such as SST $k-w$, standard $k-\varepsilon$, standard $k-w$ and RSM are used to solve numerically impact effect of the gas jet from a scramjet nozzle in full afterburner state. The generated sub-domains hybrid mesh is shown in Figure 5. The comparison of computational results with available experimental data is shown in Figure 6, which shows the velocity values at different distances from the axial center of single engine nozzle's outlet section. It can be seen that the computational results are in good agreement with the experimental data. The relative error of the computational results increases with the distance increasing, and the computational results are slightly larger than the experimental data. The predicted values of SST $k-w$ model are much closer to the experimental data by comparison, whose maximum error is $5.55 \%$. While the maximum error of standard $k-w$, standard $k-\varepsilon$ and RSM model are $7.25 \%, 8.63 \%$ and $8.94 \%$ respectively. It shows that the numerical method used in this paper is suitable for solving impact effect of the gas jet from an aircraft engine, and the SST $k$ - $w$ turbulence model can obtain higher prediction accuracy.

The expansion section (abba) of scramjet nozzle's throat makes the diameter of gas jet channel increase gradually, thus producing a series of expansion waves. The Mach number increases and the Mach angle decreases when the gas jet passes through an expansion wave, which forms an expansion wave cluster, as shown in Figure 7. The pressure of this area is less than that of the external environment, which indicates that the state is inflated, as shown in Figure 8. An inflated supersonic jet is compressed to form a contraction of the conical shock wave (bccb) after gas jet out of the nozzle, the velocity decreases, and the pressure increases. The shock wave at the top of cone disc is a Mach disc. After the conical shock wave, the direction of gas jet in the area (bcd) is parallel to the line (bc), the velocity of gas jet after the

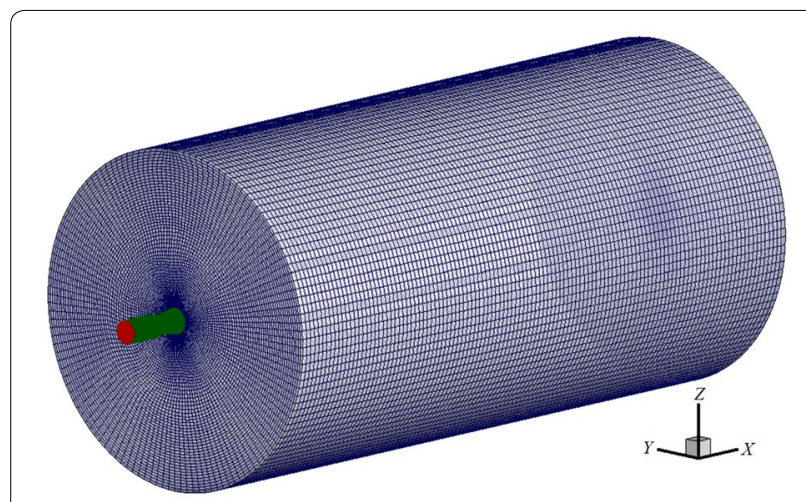

Figure 5 Mesh form of computational domain for a single engine

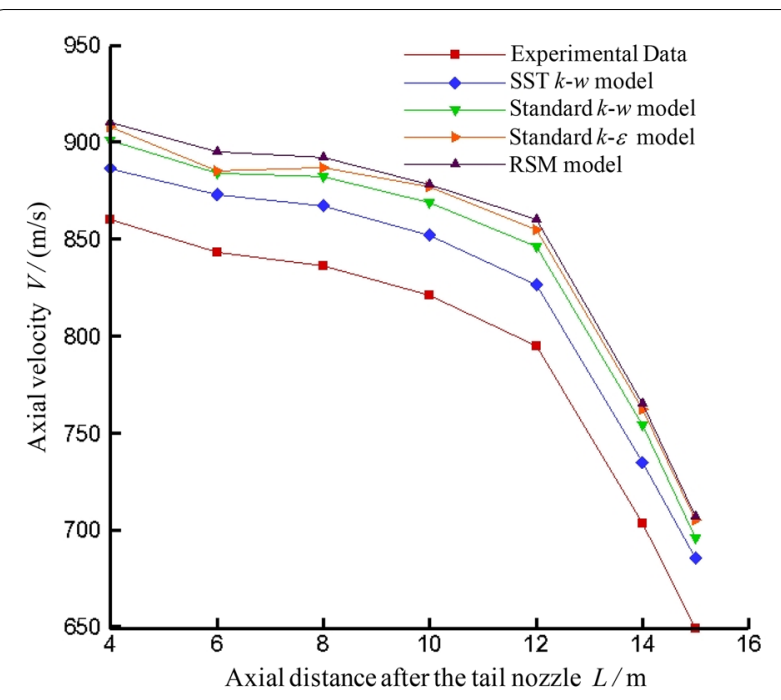

Figure 6 Axial velocity curve of gas jet from a single engine

Mach disc decreases, and the direction is parallel to the central axis, which forms an expansion of conical shock wave (cddc). The shock wave (cd) is reflected on the free boundary. Then a series of expansion waves are formed after the section (dd). The gas jet begins to expand, and it is still supersonic flow. But the pressure is higher than the environmental pressure, which leads to the formation of supersonic flow. Due to the alternation of the expansion wave and the compression wave, a series of wave and wave sections form, which make the velocity and temperature of the supersonic jet drop slowly, as shown in Figure 9. Therefore, impact force and ablation strength of the gas jet from an aircraft engine on a jet blast deflector are large, which is the main problem to be considered during the design of a jet blast deflector.

Figure 10 shows the axial static pressure, static temperature and Mach number of gas jet from the aircraft engine. Generally, the region from inlet section of scramjet nozzle to first step point of static pressure, static temperature and Mach number occurring on central axis is called initial section. The region from first step point to stable change point is called turbulent transition section.

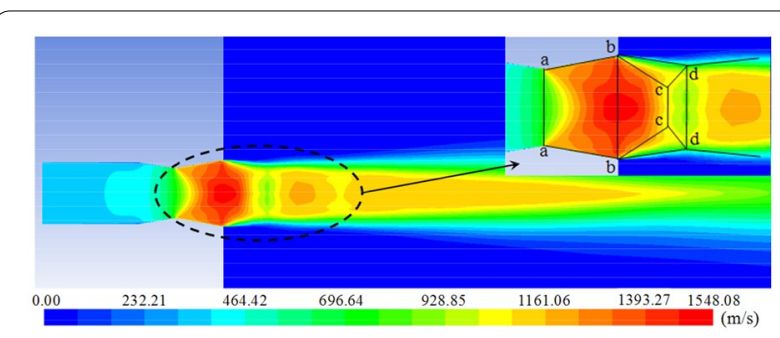

Figure 7 Velocity contour of gas jet from a single engine 


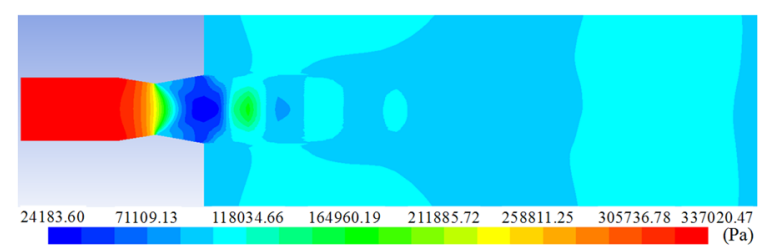

Figure 8 Static pressure contour of gas jet from a single engine

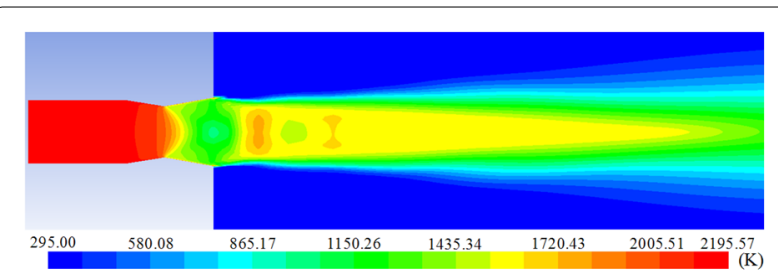

Figure 9 Static temperature contour of gas jet from a single engine

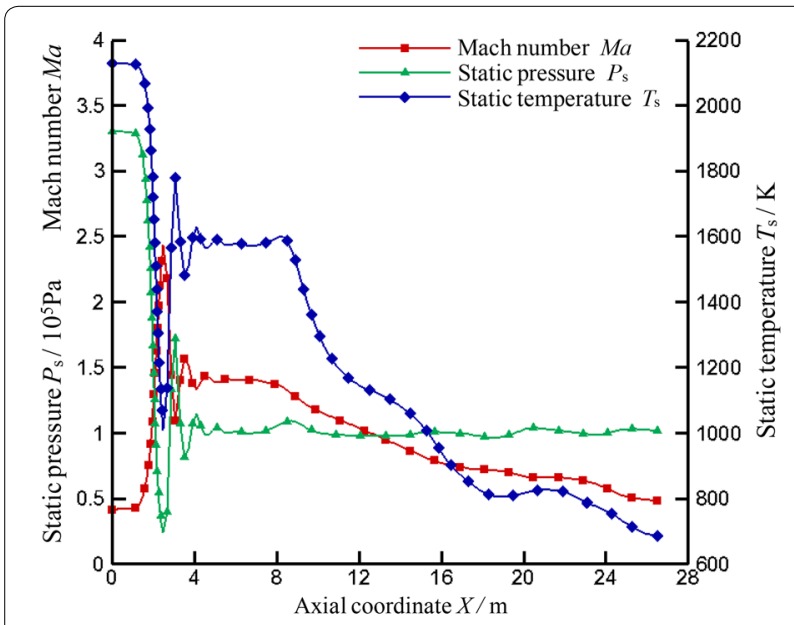

Figure 10 Axial static pressure, static temperature and Mach number curve of gas jet from the aircraft engine

The subsequent region is called turbulent full development section. Figure 10 shows that initial section, turbulent transition section and turbulent full development section are respectively $0-2.47 \mathrm{~m}, 2.47-4.68 \mathrm{~m}$ and $4.68 \mathrm{~m}-+\infty$. In the initial expansion section, static pressure, static temperature and Mach number show a monotonic change, where the static pressure and static temperature decrease rapidly, but the Mach number raises rapidly. In the turbulent transition section, due to intense changes in core area of gas jet and disintegration of shock structure, static pressure, static temperature and Mach number all change drastically. In turbulent full development section, the energy dissipation caused by mixing of gas jet and ambient air leads to static pressure decrease to the same with environmental pressure, and the static temperature and Mach number decrease continuously. After $12.68 \mathrm{~m}$, the gas jet from the aircraft engine is degenerated into subsonic flow from supersonic flow.

Figure 11 shows the axial total pressure and total temperature curves of gas jet from the aircraft engine. From the curves, it can be seen that total pressure and total temperature decrease sharply under the environmental influence at the distance of $8.50 \mathrm{~m}$ from inlet section of the scramjet nozzle. Then total pressure and total temperature decrease by half at the subsonic flow region, which provides a theoretical basis for the layout of a jet blast deflector. Usually, a jet blast deflector should be arranged at outside of the conical core region of total pressure and total temperature, so as to ensure that the kinetic and thermodynamic properties of a jet blast deflector, as shown in Figure 12 and Figure 13.

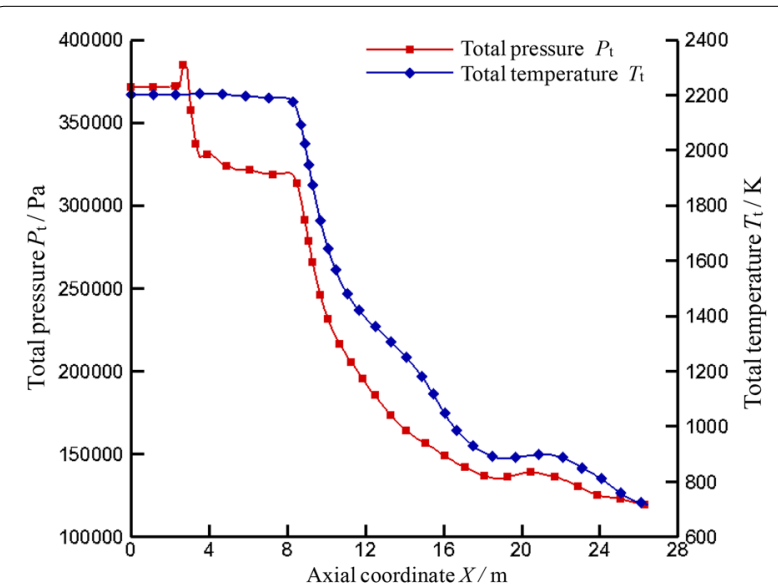

Figure 11 Axial total pressure and total temperature curve of gas jet from the aircraft engine

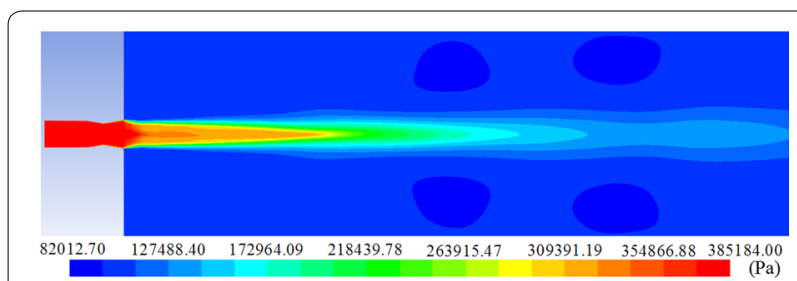

Figure 12 Total pressure contour of gas jet from a single engine

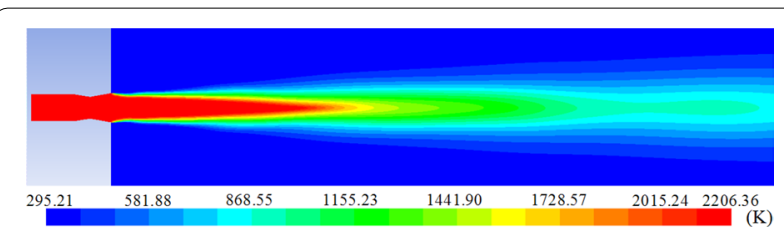

Figure 13 Total temperature contour of gas jet from a single engine 


\subsection{Impact Characteristics Analysis of Gas Jet Based on Fluid-Solid Coupling Effect}

A jet blast deflector mainly guides high temperature and high-speed gas jet from the aircraft engines overboard and upwards, in order to reduce impact influence of the gas jet on surrounding environment. Figure 14 shows static pressure distribution on the horizontal axis symmetrical surface of the gas jet from twin engines. It can be seen that the gas jet from twin engines does not interfere with each other before gas jet impacting the jet blast deflector, which has the same flow characteristics with the gas jet from a single engine. The shock wave system forms in initial section and turbulent transition section of the gas jet from each engine. The number of the wave segments is 4 . Supersonic excessive expansion flow forms in the turbulent full development section, as shown in Figure 15. Due to turning injection of the jet

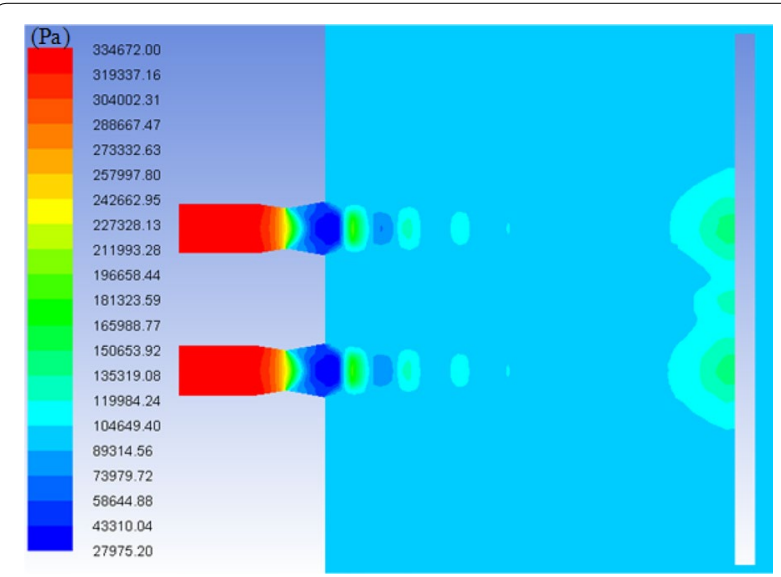

Figure 14 Static pressure contour of gas jet from twin engines

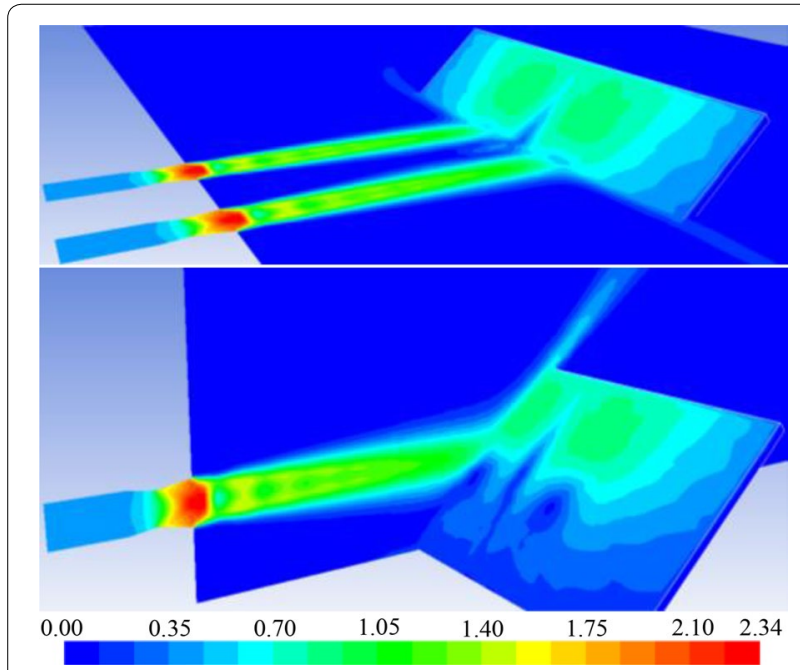

Figure 15 Mach number contour of gas jet from twin engines blast deflector and convection of the gas jet from twin engines on jet blast deflector, the local stagnation regions of two circular and a strip form on the jet blast deflector, where are the most serious impact parts of gas jet on the jet blast deflector. Diffusion and impact strength of the gas jet after being deflected is related to tilt angle of the jet blast deflector, distance from nozzle outlet to jet blast deflector and other factors.

Figure 16 shows total pressure distribution in horizontal symmetry plane and vertical symmetry plane of the gas jet, and Figure 17 shows total temperature distribution. It can be seen that the core triangle areas of total pressure and total temperature do not reach the jet blast deflector, which can significantly reduce impact force and ablation area on the jet blast deflector. High pressure area mainly concentrates on upper half of the jet blast deflector, forming dynamic impact as $X$ axis of $146077 \mathrm{~N}$ and

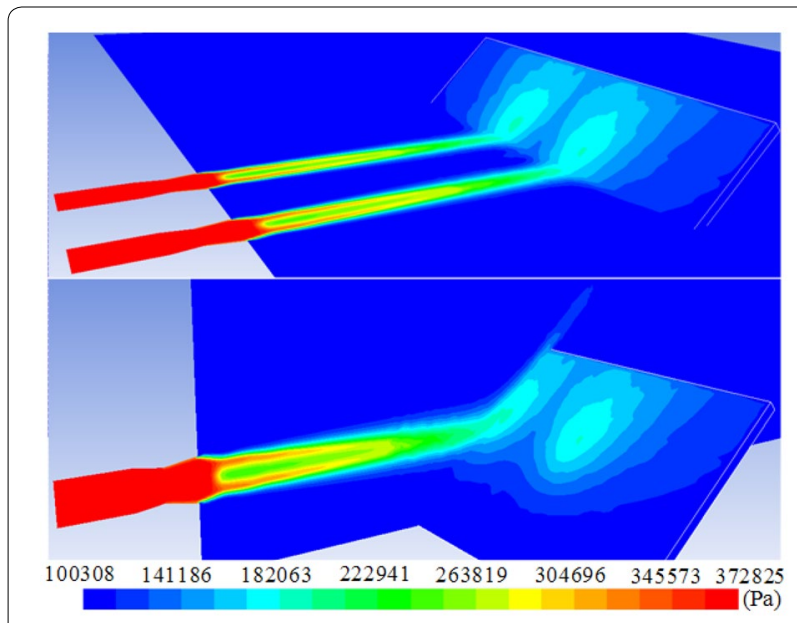

Figure 16 Total pressure contour of gas jet from twin engines

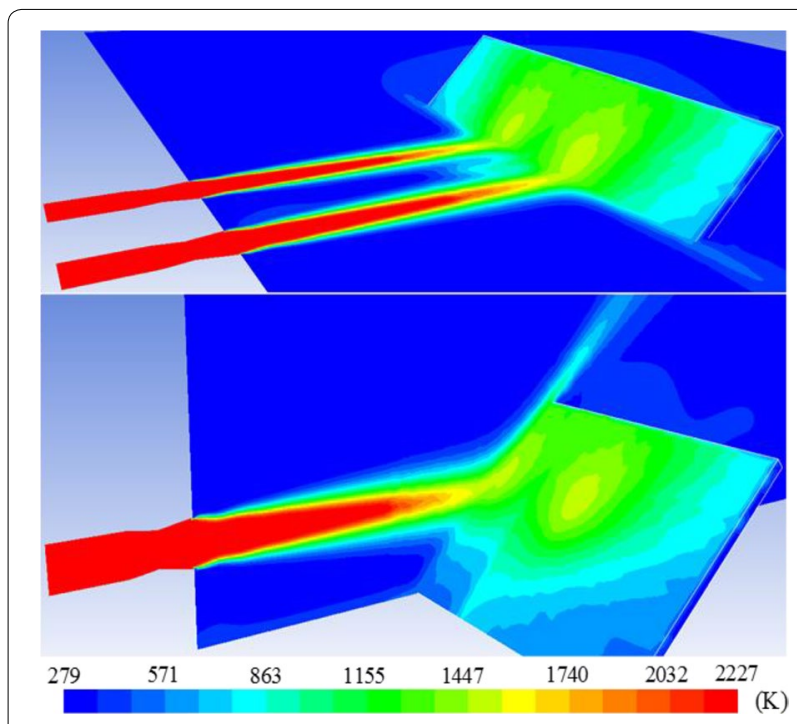

Figure 17 Total temperature contour of gas jet from twin engines 


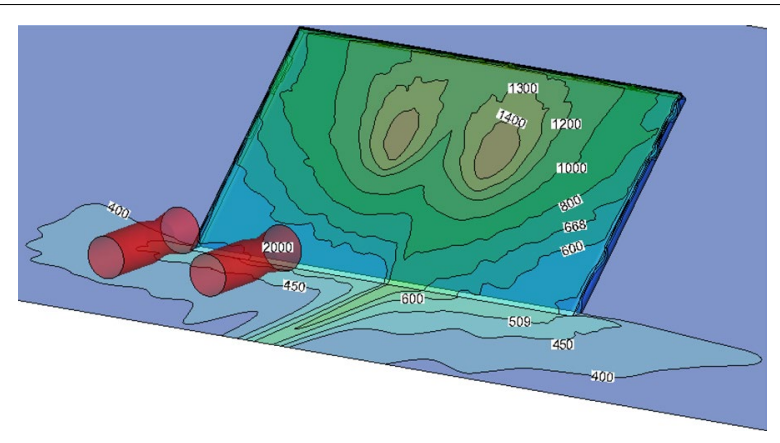

Figure 18 Total temperature contour of the jet blast deflector

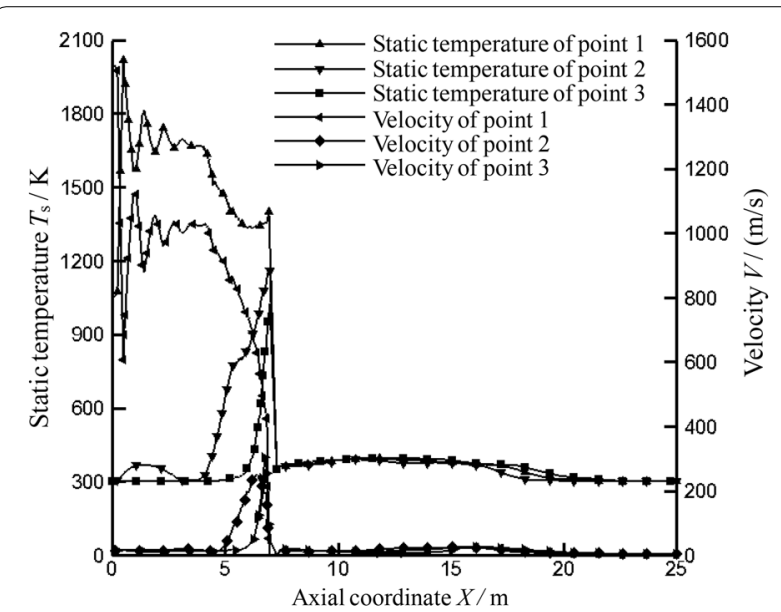

Figure 19 Static temperature and velocity curve of gas jet from twin engines

$-Y$ axis of $411957 \mathrm{~N}$, which causes a torque of 562597 $\mathrm{Nm}$ around the rod fulcrum on back of the jet blast deflector. The high-temperature areas mainly concentrate on upward 2/3 part of the jet blast deflector. The highest temperature areas are facing the twin scramjet nozzles, which produce thermal shock effect of above $1400 \mathrm{~K}$, as shown in Figure 18. In addition, due to block effect of the jet blast deflector on gas jet, the temperature of the flight deck in front of the jet blast deflector is relatively high, which will cause a threat to crew and equipments on the flight deck. So it is necessary to determine dangerous areas around the jet blast deflector.

Figure 19 shows static temperature and velocity change of three points along $X$ axis, including center of left engine (Point 1), midpoint of center line between twin engines (Point 2) and isometric point of left engine (Point 3). Due to the existence of shock wave, the initial stage of Point 1's static temperature and velocity is a decay pulse curve. With the distance from scramjet nozzle increasing, the static temperature and velocity decay slowly. After the jet blast deflector, static temperature and velocity drop to the same parameters with surrounding environment. The temperature and velocity of Point 2 and Point 3 only increase suddenly in front of the jet blast deflector, which are the same parameters with surrounding environment in other areas. Due to interaction of the gas jet from twin engines on the jet blast deflector, the temperature and velocity of Point 2 increase faster than that of Point 3.

High-temperature gas jet from aircraft engines is easy to make crew on the flight deck burned after it impacting the jet blast deflector. According to medical experiments, it is difficult to breathe and endure in the temperature of more than $116{ }^{\circ} \mathrm{C}$ [25]. Figure 20 shows static temperature contour surface of $116^{\circ} \mathrm{C}$ in the whole computational domain. It can be seen that there are hightemperature areas at both sides and front of the jet blast deflector, high-temperature areas exist at both sides of the jet blast deflector's rear because of gas vortex, while the right rear is relatively safe. The carrier aircrafts usually can withstand the maximum temperature of $150{ }^{\circ} \mathrm{C}$. Figure 21 shows static temperature contour surface of $150{ }^{\circ} \mathrm{C}$ in whole computational domain. It can be seen that the carrier aircrafts parking at rear of the jet blast deflector are absolutely safe. According to the computational results, the dangerous area of high-temperature gas jet for crew on the flight deck is that $X$ axis from $0 \mathrm{~m}$ to $16.2 \mathrm{~m}$ and $Z$ axis from $6.8 \mathrm{~m}$ to $29.2 \mathrm{~m}$.

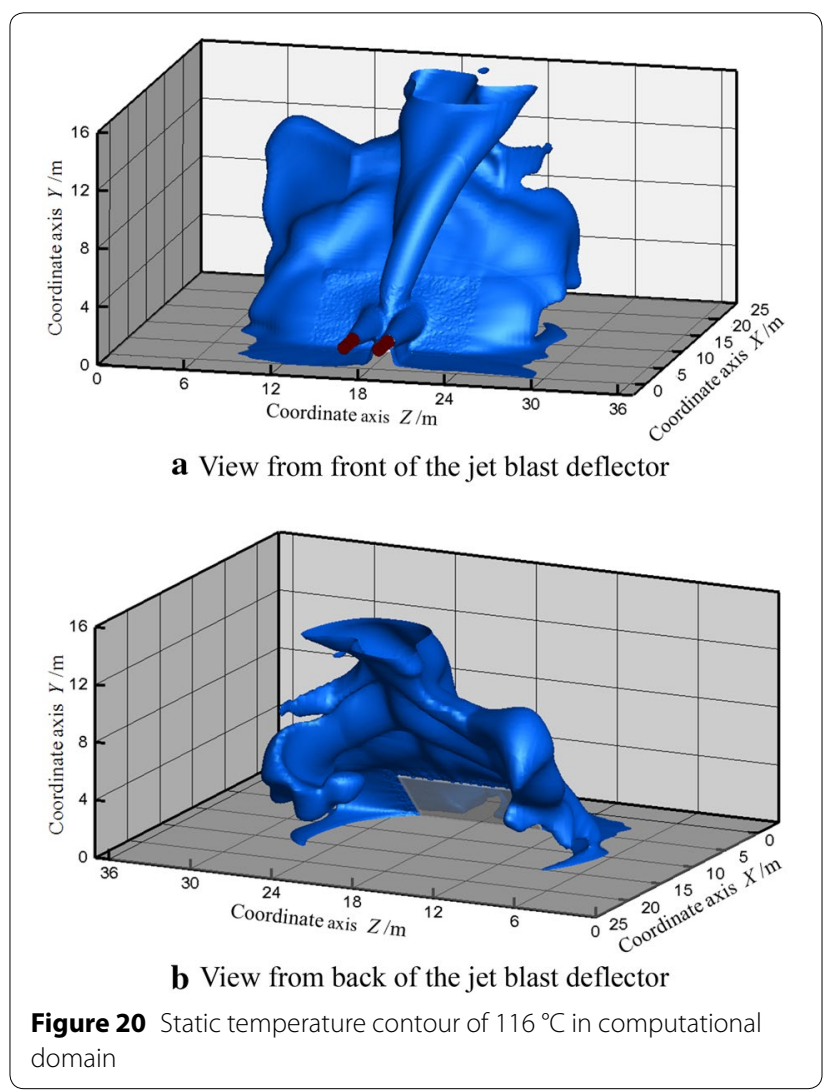




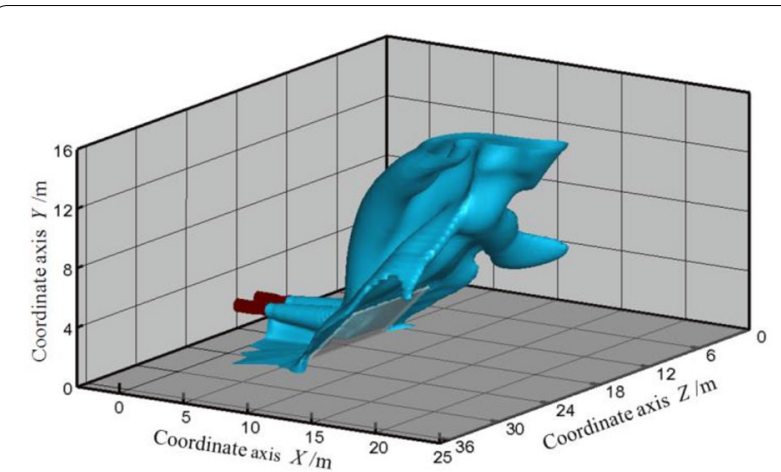

Figure 21 Static temperature contour of $150^{\circ} \mathrm{C}$ in computational domain

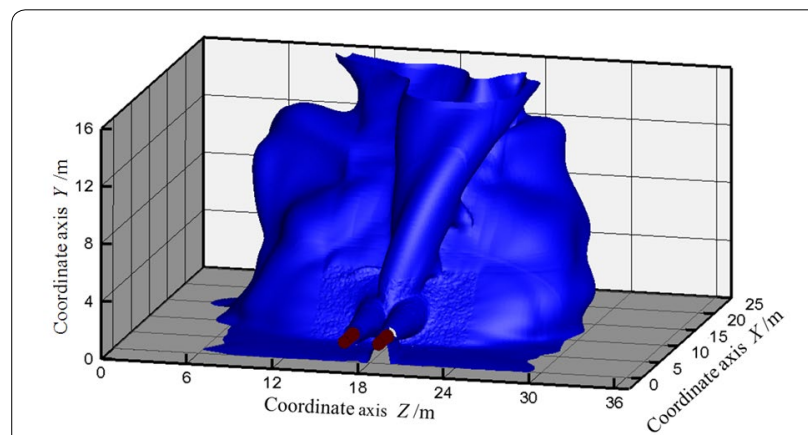

a View from front of the jet blast deflector

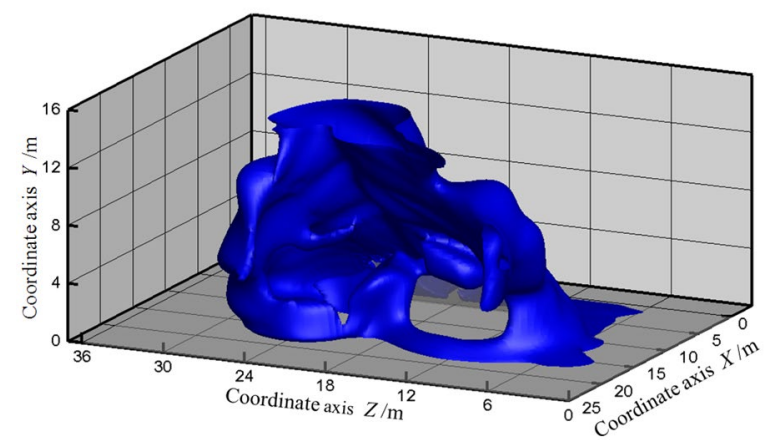

b View from back of the jet blast deflector

Figure $\mathbf{2 2}$ Velocity contour of $20.8 \mathrm{~m} / \mathrm{s}$ in computational domain

High-speed gas jet from aircraft engines is easy to blow away crew on the flight deck after it impacting the jet blast deflector. According to meteorological data, humans will be blown away at the wind speed of higher than force 9, and the force 9 wind speed is $20.8 \mathrm{~m} / \mathrm{s}$ [26]. Figure 22 shows the velocity contour surface of $20.8 \mathrm{~m} / \mathrm{s}$ in computational domain. It can be seen that there are high-speed areas at both sides, front and rear of the jet blast deflector, where highspeed eddy current with large radius exists at rear of

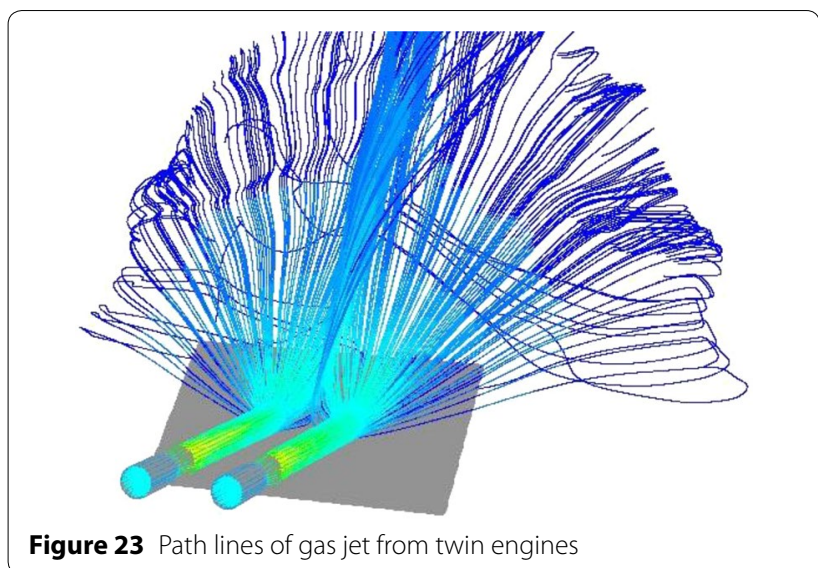

Figure $\mathbf{2 3}$ Path lines of gas jet from twin engines

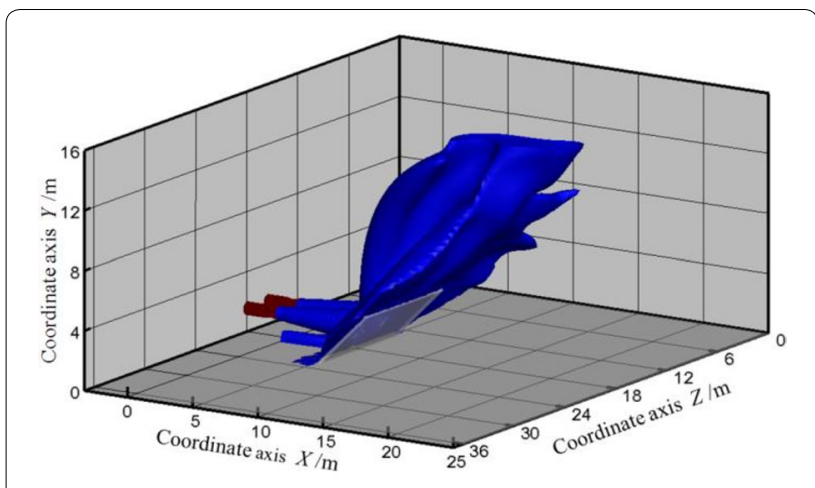

Figure 24 Velocity contour of $51 \mathrm{~m} / \mathrm{s}$ in computational domain

the jet blast deflector, as shown in Figure 23. This is because the local low-pressure area caused by flow velocity difference behind the jet blast deflector forms a vortex core. The carrier aircrafts usually can withstand the maximum typhoon of level 16, and the speed of typhoon level 16 is $51 \mathrm{~m} / \mathrm{s}$. Figure 24 shows the velocity contour surface of $51 \mathrm{~m} / \mathrm{s}$ in whole computational domain. It can be seen that the carrier aircrafts parking at rear of the jet blast deflector will not be blown away. According to the computational results, the dangerous area of high-speed gas jet for crew on the flight deck is that $X$ axis from $0 \mathrm{~m}$ to $18.9 \mathrm{~m}$ and $Z$ axis from $4.6 \mathrm{~m}$ to $29.6 \mathrm{~m}$.

In short, the jet blast deflector can well deflect highspeed, high-pressure and high-temperature gas jet, and main harm of the gas jet from twin engines on crew and equipments on the flight deck is greatly weakened. According to the dangerous area of high-temperature and high-speed gas jet, it is recommended to determine the dangerous area of gas jet as $X$ axis from $0 \mathrm{~m}$ to 19.8 $\mathrm{m}$ and $Z$ axis from $4.0 \mathrm{~m}$ to $30.2 \mathrm{~m}$ based on the safety factor of $5 \%$. It can ensure that crew and equipments 
outside this rectangular area on the flight deck will not be affected by the gas jet.

\section{Conclusions}

(1) In the pre-processing of numerical simulation of the supersonic gas jet from aircraft engines, the sub-domains hybrid meshing scheme applied can adapt to the complex geometry of the jet blast deflector, not only reducing the mesh number, but also improving the ability to capture complex wave system interference with higher mesh accuracy.

(2) In numerical computation of the supersonic gas jet from a single aircraft engine, the good agreement between computational results with four different turbulence models and experimental data validates the correctness of the numerical method and shows that SST $k-w$ turbulence model is more suitable for the numerical simulation of compressible viscous gas jet with high prediction accuracy.

(3) The formation and evolution of shock wave and the parameters such as velocity, pressure and temperature are got through the numerical analysis of the gas jet from a single aircraft engine, which provides a theoretical basis for layout of the jet blast deflector.

(4) The numerical method of fluid-solid coupling problem that the gas jet from aircraft engines impacting a jet blast deflector is explored out with CFD technique. The dangerous activity area of crew and equipments on the flight deck is predicted qualitatively and quantitatively based on the thermal shock properties and dynamic impact characteristics.

\section{Authors' Contributions}

F-DG was in charge of the whole trial; F-DG wrote the manuscript; D-XW, $\mathrm{H}-\mathrm{DW}$ and M-MJ assisted with sampling and laboratory analyses. All authors read and approved the final manuscript.

\footnotetext{
Authors' Information

Fu-Dong Gao, born in 1982, is currently a lecturer at Department of Carrier Aviation Security and Station Management, Naval Aeronautical University, China. He received his PhD degree from National University of Defense Technology, China, in 2012. His research interests include computational fluid dynamics, design and performance prediction of the fluid machinery.

De-Xin Wang, born in 1968, is currently an associate professor at Department of Carrier Aviation Security and Station Management, Naval Aeronautical University, China. He received his master degree on electrical engineering and automation from Beihang University, China, in 1993.

Hai-Dong Wang, born in 1974, is currently an associate professor at Depart ment of Carrier Aviation Security and Station Management, Naval Aeronautical University, China.

Ming-Ming Jia, born in 1982, is currently a lecturer at Department of Carrier Aviation Security and Station Management, Naval Aeronautical University, China.
}

\section{Competing Interests}

The authors declare that they have no competing interests.

\section{Funding}

Supported by National Natural Science Foundation of China (Grant No. 51505491), and Shandong Provincial Natural Science Foundation of China (Grant No. ZR2014EEP019).

\section{Publisher's Note}

Springer Nature remains neutral with regard to jurisdictional claims in published maps and institutional affiliations.

Received: 17 December 2016 Accepted: 21 September 2018

Published online: 11 October 2018

\section{References}

[1] LP Zhao. Numerical simulation for high temperature and high pressure flow field of aircraft engine jet impingement. Ship Science and Technology, 2016, 38(1): 145-149.

[2] K Z Yue, L L Cheng, H Liu, et al. Analysis of jet blast impact of embarked aircraft on deck takeoff zone. Aerospace Science \& Technology, 2015, 45(5): 60-66.

[3] N A Buchmann, C Atkinson, J Soria. Ultra-high-speed tomographic digital holographic velocimetry in supersonic particle-laden jet flows. Measurement Science \& Technology, 2012, 24(2): 827-837.

[4] D R Brooks, T Ecker, KT Lowe, et al. Experimental Reynolds stress spectra in hot supersonic round jets. 52nd Aerospace Sciences Meeting, Maryland, USA, January 13-17, 2014: 1227-1245

[5] S Huang, CWang, J Hu. Research on velocity field of carrier-based aircraft engine's jet flow. Journal of Harbin Engineering University, 2009, 30(4): 353-356. (in Chinese)

[6] ZY Ma, M Chu, XXu. One-dimensional performance calculation of dual mode scramjet combustor based on flamelets and jet model. Journal of Propulsion Technology, 2015, 36(1): 104-111. (in Chinese)

[7] L HWang, Z W Wu, HW Chi, et al. A method of quasi-one dimensional numerical analysis for solid fuel scramjet combustor performance. Journal of Solid Rocket Technology, 2013, 36(6): 742-747. (in Chinese)

[8] M A Pakhomov, V I Terekhov. RANS modeling of flow structure and turbulent heat transfer in pulsed gas-droplet mist jet impingement. International Journal of Thermal Sciences, 2016, 100(2): 284-297.

[9] L Lin, C S Weng. Two-dimensional numerical calculation for the influence of plasma jet ignition on deflagration-to-detonation transition. Acta Armamentarii, 2014, 35(9): 1428-1435. (in Chinese)

[10] M Yu, Y H Liu. Numerical simulation on combination flow field for axisymmetric convergent divergent nozzle. Science Technology and Engineering, 2011, 11(32): 7979-7984. (in Chinese)

[11] A Melaibari, P Molian, P Shrotriya. Two-dimensional contour cutting of polycrystalline cubic boron nitride using a novel laser/water jet hybrid process. The International Journal of Advanced Manufacturing Technology, 2012, 63(5): 641-649.

[12] KXu. Research of adaption between aircraft and jet blast deflector. Harbin: Harbin Engineering University, 2011. (in Chinese)

[13] R Paoli, L Nybelen, J Picot, et al. Effects of jet/vortex interaction on contrail formation in supersaturated conditions. Physics of Fluids, 2013, 25(5): $25-30$

[14] ANSYS INC. ANSYS FLUENT user's guide. Shanghai: ANSYS Inc, 2011.

[15] M Shives, C Crawford. Adapted two-equation turbulence closures for actuator disk RANS simulations of wind \& tidal turbine wakes. Renewable Energy, 2016, 92: 273-292.

[16] W R Zhu, Z X Gao, Y J Tang, et al. Adaptability of turbulence models to predict the performance and blade surface pressure prediction of a Francis turbine. Engineering Computations, 2015, 33(1): 238-251.

[17] M Nataraj, R R Singh. Analyzing pump impeller for performance evaluation using RSM and CFD. Desalination \& Water Treatment, 2014, 52(34): 6822-6831

[18] CWang. The influence of aircraft engine's jet flow around ship deck. Harbin: Harbin Engineering University, 2007. (in Chinese) 
[19] Q F Zhang, P P Yan, W M Gao, et al. Influence of jet blast deflector backflow on inlet temperature. Aeronautical Computing Technique, 2016, 46(4): 35-38. (in Chinese)

[20] P Anoop, C Unnikrishnan, B Sundar, et al. Thermal analysis of a jet deflector subjected to liquid engine jet exhaust in a static test. Heat Transfer Engineering, 2015, 36(4): 346-351.

[21] F D Gao, CY Pan, X J Xu, et al. Numerical computation and analysis of high-speed autonomous underwater vehicle (AUV) moving in head sea based on dynamic mesh. Journal of Central South University, 2012, 19(11): 3084-3093.

[22] X M Zhou, Z K Wang, Y F Zhang. Grid-Adaption Based CFD Method for the Flow Coefficient Calculation of Valves. Journal of University of Electronic Science and Technology of China, 2017, 46(2): 475-480.
[23] A Lopez, W Nicholls, M T Stickland, et al. CFD study of jet impingement test erosion using Ansys Fluent. Computer Physics Communications, 2015, 197(6): 88-95.

[24] R M Coroneos, R S R Gorla. Structural analysis and optimization of a composite fan blade for future aircraft engine. International Journal of Turbo \& Jet-Engines, 2016, 29(3): 131-164.

[25] M J Buller, W J Tharion, S N Cheuvront, et al. Estimation of human core temperature from sequential heart rate observations. Physiological Measurement, 2013, 34(7): 781-798.

[26] T Stathopoulos. Pedestrian level winds and outdoor human comfort. Journal of Wind Engineering and Industrial Aerodynamics, 2006, 94(11): 769-780.

\section{Submit your manuscript to a SpringerOpen ${ }^{\circ}$ journal and benefit from:}

- Convenient online submission

- Rigorous peer review

- Open access: articles freely available online

- High visibility within the field

- Retaining the copyright to your article

Submit your next manuscript at $\boldsymbol{\nabla}$ springeropen.com 